\title{
Current and future strategies for the treatment of chronic hepatitis C
}

\author{
Omar Alshuwaykh and Paul Y. Kwo \\ Department of Medicine, Stanford University School of Medicine, Stanford, CA, USA
}

Chronic hepatitis $C$ infection is a major cause of liver disease and hepatocellular carcinoma worldwide. While hepatitis $C$ has been treated for decades with some success, the introduction of direct acting antiviral agents has revolutionized the treatment of hepatitis $\mathrm{C}$ with finite, highly effective, well-tolerated therapy and there are few populations that cannot be successfully treated now or are complicated to manage. The World Health Organization has released elimination targets in an effort to eliminate viral hepatitis and reduce dramatically the morbidity and mortality caused by both viral hepatitis. While hepatitis $C$ is straightforward to treat, it remains problematic to eliminate on a global scale. Diagnosis of hepatitis $\mathrm{C}$ remains the major gap in the cascade of care and numerous screening strategies will be required to reduce this gap. While historically, treatment of hepatitis $C$ has been centralized, decentralized approaches will be required to diagnose, evaluate, and link to care the large population of individuals worldwide with hepatitis $\mathrm{C}$ across low-, middle-, and high-income countries. With the introduction of multiple pangenotypic treatment options and reduced cost for these therapies, assessment and treatment for those with hepatitis $C$ has been simplified and made more accessible worldwide. There are multiple populations for whom care models are being developed and refined, including those when inject drugs, those who are incarcerated, those who present with sexually transmitted disease including the men who have sex with men population, amongst many others. While a vaccine for hepatitis $C$ remains elusive these efforts continue. Multiple successful elimination efforts have been reported. (Clin Mol Hepatol 2021;27:246-256)

Keywords: Hepatitis C; Antiviral agents; Disease eradication; Substance abuse; Intravenous

\section{INTRODUCTION}

Since the identification of hepatitis C cDNA clone along with an assay for circulating antibodies in 1989, few diseases have undergone such a rapid advance in diagnostics and therapeutics as has the treatment of chronic hepatitis $\mathrm{C}^{1,2} \mathrm{~A}$ transmissible agent caus- ing viral hepatitis was proposed in 1951 with multiple subsequent reports recognizing that serum hepatitis occurred in persons who injected drugs (PWID). ${ }^{3}$ Prior to the identification of the hepatitis $C$ virus (HCV) it was recognized that those with non-A non-B hepatitis who received a course of interferon would normalize their liver tests. With the identification of the HCV, and improved

\begin{abstract}
Abbreviations:
APASL, Asian Pacific Association for the Study of the Liver; APRI, aspartate aminotransferase-platelet index; DAAs, direct acting antiviral agents; ECHO, Extension for Community Healthcare Outcomes; FIB-4, fibrosis-4; HBV, hepatitis $B$ virus; HCV, hepatitis C virus; HIV, human immunodeficiency virus; MSM, men who have sex with men; OST, opiate substitution therapy; PCR, polymerase chain reaction; PrEP, pre-exposure prophylaxis; PWID, persons who injected drugs; SVR, sustained virologic response; WHO, World Health Organization
\end{abstract}

\section{Corresponding author : Paul Y. Kwo}

Department of Medicine, Stanford University School of Medicine, 430 Broadway, Pavilion C. 3rd Floor Redwood City, CA 94063, USA

Tel: +1-650-723-7040, Fax: +1-650-498-5692

E-mail: pkwo@stanford.edu

https://orcid.org/0000-0002-8234-4485 
detection of hepatitis C RNA in serum, it was recognized that interferon-based therapy could lead to sustained clearance of hepatitis $C$ from the serum. ${ }^{4}$ The discovery of hepatitis $C$ genotypes allowed clinicians to further tailor their approach to treatment of hepatitis $C$, with the addition of ribavirin improving sustained response rates while adding to the hepatitis $\mathrm{C}$ treatment side effect profile. ${ }^{5}$ The identification and characterization of the nonstructural replication proteins of the HCV allowed for targeting with inhibitors of specific replication pathway proteins including the NS3 protease, the NS5a replication complex, and the NS5a polymerase. ${ }^{6}$ The introduction of all oral direct acting antiviral agents (DAAs) has revolutionized the treatment of those with chronic hepatitis $C$. Rather than endure a difficult course of therapy that may last 6 to 12 months, treatment of hepatitis C typically lasts 8 to 12 weeks with sustained response or cure rates well over $90 \%$. In this review, we will discuss the current approach to treatment of hepatitis $C$ as well as how current and future strategies will need to adapt to the evolving epidemiology of hepatitis $C$ infection to ensure improved rates of diagnosis, linkage to care, and treatment. Improved access to therapy, with simplified diagnostics and treatment algorithms can provide a framework to allow countries and regions of the world to initiate plans for successful treatment and elimination of hepatitis $\mathrm{C}$.

Chronic HCV infection is a global health problem, and a leading cause of chronic liver disease and hepatocellular carcinoma. Worldwide, 71.1 million individuals are estimated to have chronic hepatitis C infection with active viremia and in 2015 there was an estimated 580,000 people deaths from cirrhosis and liver cancer. ${ }^{7}$ It is estimated that $80 \%$ of all cases of HCV occur in 31 countries, with China, Pakistan, India, Egypt, Russia, and the USA accounting for more than $50 \%$ of all HCV infections globally. The introduction of the highly effective and tolerable DAAs led the World Health Organization (WHO) in 2016 to call for hepatitis B and C elimination by 2030 , with goals of a $90 \%$ reduction in incident cases of hepatitis $B$ and $C$ and a $65 \%$ reduction in mortality from viral hepatitis. ${ }^{8}$ Currently it is estimated that less than $20 \%$ of individuals with HCV have been diagnosed and only $7 \%$ of those diagnosed have initiated treatment with only 12 countries reported to be on track at present to reach the WHO goal. ${ }^{9}$

\section{CHANGING EPIDEMIOLOGY OF HEPATITIS C}

There have been changes in the epidemiology of hepatitis $C$ in some parts of the world with the opioid epidemic resulting in an increase in reported acute $\mathrm{HCV}$, particularly in those who are younger. ${ }^{10,11}$ Worldwide, there may be up 10 million PWIDs with an estimated $2 / 3$ of these individuals being infected with $\mathrm{HCV}^{12}$ As a result of the opioid epidemic, in certain countries there is now a bimodal age distribution of HCV reflecting a higher prevalence of infection in both younger (aged 20-40 years) and older (aged $>50$ years) individuals with injection drug use now the leading cause of new infections in the younger population including women of child-bearing age. ${ }^{13}$ In addition, there are approximately 2.3 million individuals co-infected with HCV and human immunodeficiency virus (HIV), with higher prevalence in men who have sex with men (MSM) and in injection drug users. It is also estimated that there are approximately 10 million individuals who are currently incarcerated and the global prevalence of HCV in the incarcerated population has recently been estimated at $15.1 \%$. Diagnosis and treatment models for these populations must also be constructed as a part of a global elimination strategy. 14,15

Current care models to treat HCV have historically applied a centralized approach. Individuals are diagnosed with HCV, and are then referred to specialists for further evaluation, staging of liver disease and initiation of treatment. This paradigm evolved from the complexities of the prior interferon-based therapies and continued through the early stages of the introduction of DAAs. This was due to many factors including the expense of initial DAA treatments, drug interactions, and the initial large group of individuals with decompensated liver disease that required close monitoring during treatment. Many of these individuals who were already in engaged in care were successfully treated and in high income parts of the world, successful treatment of HCV has led to reductions in listings for transplant for hepatitis C-related cirrhosis. This paradigm continues in many parts of the world. ${ }^{16} \mathrm{Howev}-$ er, there are not enough specialists available to care for the large number of HCV-infected individuals worldwide.

\section{Cascade of care for hepatitis C}

The current cascade of care for HCV involves multiple steps (Fig. 1). Diagnosis of HCV remains the largest gap in the cascade of care. Current diagnostic testing is based on serologic assays to detect anti-HCV antibodies, but this requires confirmation of active infection, typically by confirming the presence of HCV RNA. Hepatitis $C$ screening has historically been based on risk behaviors, risk exposures, as well as being part of the evaluation of those with abnormal liver chemistries. Risk-based screening has been shown to be highly effective in many countries who have universal access 


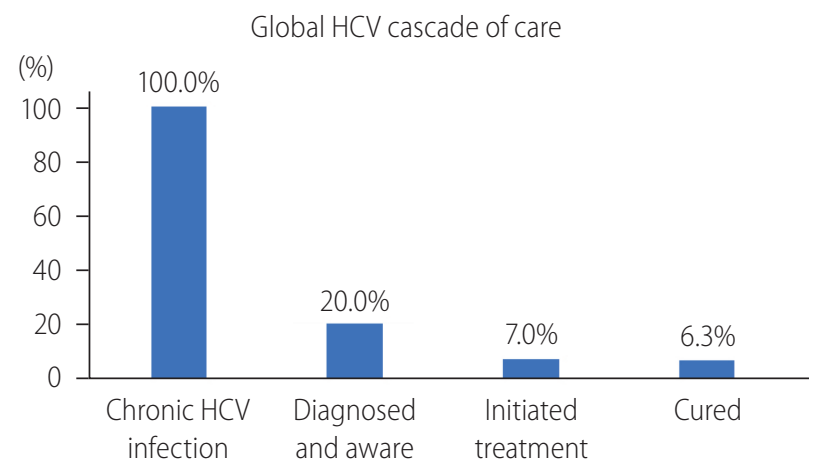

Figure 1. The cascade of care in hepatitis $C$. The diagnosis of hepatitis $C$ remains the largest gap in the cascade of care. Adapted from Lazarus et al. ${ }^{9} \mathrm{HCV}$, hepatitis $\mathrm{C}$ virus.

to healthcare and defined national strategies to eliminate $\mathrm{HCV}$, including universal access to treatment for HCV. As an example, Australia, one of the countries currently on track to meet WHO guidance for HCV elimination has employed a risk-based strategy along with other measures to achieve a diagnosis rate of approximately $80 \% .{ }^{17}$ Other effective strategies could include universal prenatal screening, screening for those who are incarcerated, screening those presenting for treatment for opioid use disorder, and screening for HCV in emergency departments and during hospitalizations. In the USA, recent societal and governmental recommendations for universal screening have been added for those who are between ages 18 to 70 years of age..$^{18,19}$ These changes were in response to the increasing prevalence of $\mathrm{HCV}$ in those between the ages of 18 and 40 which has been driven by the opiate epidemic. The European Association for the Study of Liver Disease 2018 practice guidance recommends HCV screening for the general population according to the local epidemiology of $\mathrm{HCV}$ infection, with testing in areas of intermediate to high seroprevalence ( $\geq 2 \%-5 \%$ ), populations at risk of HCV infection, and birth cohort testing and similarly, the Asian Pacific Association for the Study of the Liver (APASL) 2016 practice guidance also recommends HCV screening based on the geographical region and high-risk populations including PWID and MSM. ${ }^{20,21}$ The WHO 2017 practice guidance recommends HCV screening for the general population in high HCV prevalence countries and for individuals with high risk behaviors including PWID, MSM, prisoners, HIVinfected persons and commercial sex workers, and birth cohort testing. ${ }^{22}$ Moreover, in high risk populations that are successfully treated, regular screening protocols for recurrent infection are required along with efforts for harm reduction. A combination of risk-based screening strategies and universal screening strategies in areas where risk-based screenings have not been effective will be required to diagnose the large number of individuals who are unaware of their infection.

\section{Diagnosis and staging of hepatitis $C$}

There are widely available serologic screening tests for $\mathrm{HCV}$ and for low, middle countries and high income countries with serum and point-of-care testing being available at costs as low as 1 USD for low and middle income countries (Table 1). Active viremia is confirmed with polymerase chain reaction (PCR) testing and can be a costly step in the treatment of HCV with the cost of confirming viremia approaching the cost of hepatitis $C$ treatment in some parts of the world. Moreover, point of care testing to confirm viremia remains a logistical challenge and alternatives such as measurement of HCV core antigen should be explored. ${ }^{23}$ Historically, determining the genotype has been part of the standard of care in the treatment of hepatitis $\mathrm{C}$ and was required to choose or exclude a specific therapy or to determine if additional therapies such as ribavirin were required. A genotype is only required if required to make treatment decisions if pangenotypic therapy is not available or if specified by the payor.

Assessment of fibrosis has been essential in the evaluation of those with HCV. Those with cirrhosis are at increased risk for hepatocellular carcinoma, and require additional care including monitoring for decompensation, screening for hepatocellular carcinoma, and an assessment for the presence or absence of esophageal varices. While resource limited countries may have limited access to subspecialty services to care for those with cirrhosis, fibrosis assessment remains an important part of the evaluation of those with chronic hepatitis $C$ infection. Noninvasive assessment of fibrosis has replaced liver biopsy with noninvasive tests of fibrosis including but not limited to elastography, aspartate aminotransferase-platelet index (APRI), and fibrosis-4 (FIB-4) with the latter 2 being used frequently in resource constrained countries. There are a variety of commercial panels and other technologies that are available to estimate the level of fibrosis in countries that are not resource constrained (Table 2).

\section{Simplified treatment for hepatitis $C$}

Once viremia has been confirmed, those with HCV who can comply with therapy are offered treatment with DAA therapy. In the past, many populations were excluded from treatment for hepatitis $\mathrm{C}$ due to side effects of prior interferon-based therapies. 
Table 1. World Health Organization pre-qualified diagnostic tests

\begin{tabular}{llll}
\hline \multicolumn{1}{c}{ Product } & \multicolumn{1}{c}{ Manufacturer } & Technology \\
\hline HCV test & SD Bioline & Standard Diagnostic Korea & RDT (Ab) \\
& OraQuick & OraSure & RDT (Ab) \\
& Rapid Anti-HCV & RDT (Ab) \\
& STANDARD Q HCV Ab & SD Biosensor & RDT (Ab) \\
Confirmatory test & GeneXpert & Cepheid & Near-POC \\
& Genedrive & Genedrive & Near-POC \\
& Truelab/Truenat & POC \\
Architect & Molbio Diagnostics & NAT \\
Aptima & Abbott & NAT \\
CAP/CTM HCV & Hologic & NAT \\
Versant HCV & Roche & SAT \\
Truelab/Truenat & Siemens & POC \\
Real Time & Abbott & Biocentric & NAT \\
Generic & Hologic & Qiagen & NAT \\
Aptima & Siemens & NAT \\
Artus HCV & Versant HCV & NAT & NAT
\end{tabular}

Adapted from World Health Organization. ${ }^{47,48}$

RDT, rapid diagnostic test; $A b$, antibody; HCV, hepatitis C virus; POC, point of care; NAT, nucleic acid test.

At present, the difficult to treat populations are few with high sustained virologic response (SVR) rates being achieved in those treated with DAAs in cirrhosis patients, HIV/HCV co-infection, renal disease, and transplant populations. Moreover, acute hepatitis $C$ infection can be treated effectively with short duration DAA therapy and modeling studies have suggested it is cost saving to treat all individuals who present with acute hepatitis $C .{ }^{24}$ The current most widely available DAA therapies worldwide may be divided into pangenotypic and genotype specific therapies (Table 3). There are now three widely available first line pangenotypic therapies approved for the treatment of hepatitis $\mathrm{C}$ in addition to a salvage regimen that is used for those who fail to achieve sustained response. Drug-drug interactions must be assessed prior to initiation of therapy but there are relatively few medicines that constitute absolute contraindications to administration of DAA therapy where an alternative regimen cannot be found.

Monitoring of those on treatment for HCV has also been simplified greatly. Previously liver tests, renal function, complete blood count, and viral levels were monitored during treatment for safety and efficacy. The currently available approved DAAs have high efficacy and wide safety margins with few exceptions and monitoring may be tailored to those with significant drug interactions, co-
Table 2. Fibrosis assessment tools before initiating HCV therapy

\begin{tabular}{ll}
\hline LMICs & \multicolumn{1}{c}{ HICs } \\
\hline Fibrosis-4 & All LMICs tools \\
$\begin{array}{l}\text { Aspartate aminotransferase- } \\
\text { platelets ratio }\end{array}$ & FibroScan \\
Platelet count & Elastography \\
& US/CT/MRI \\
& Liver biopsy \\
& Commercial serum markers \\
& Enhanced liver fibrosis panel \\
& Hepascore \\
\hline
\end{tabular}

HCV, hepatitis C virus; LMICs, low to middle income countries; HICs, high income countries; US, ultrasound; CT, computed tomography; MRI, magnetic resonance imaging.

infection with other viruses, and those with cirrhosis. Recent practice guidance recommendations and expert opinion have advocated for simplified treatment algorithms with pangenotypic therapy, with minimal monitoring during treatment with confirmation of SVR 12 to 24 weeks after completion of therapy. ${ }^{25,26}$ These emerging simplified treatment strategies will be especially useful during the COVID 19 pandemic where there will be challenges in 
Table 3. Major first line DAA treatment options

\begin{tabular}{|c|c|c|c|c|}
\hline \multirow{2}{*}{$\begin{array}{l}\text { HCV genotype } \\
1 / 4\end{array}$} & \multicolumn{2}{|c|}{ No cirrhosis } & \multicolumn{2}{|c|}{ Compensated cirrhosis } \\
\hline & GLE/PIB* & $8 W$ & GLE/PIB* & $8 \mathrm{~W}$ \\
\hline & SOF/VEL $L^{*}$ & $12 \mathrm{~W}$ & SOF/VEL & $12 W$ \\
\hline & SOF/DAC* & $12 \mathrm{~W}$ & SOF/DAC* & $12-24 \mathrm{~W}$ \\
\hline & LDV/SOF & $8-12 W$ & LDV/SOF & $12 \mathrm{~W}$ \\
\hline & EBR/GZR & $12 \mathrm{~W}$ & EBR/GZR & $12 W$ \\
\hline \multirow[t]{3}{*}{2} & GLE/PIB* & $8 W$ & GLE/PIB* & $8 W$ \\
\hline & SOF/VEL $L^{*}$ & $12 \mathrm{~W}$ & SOF/VEL & $12 W$ \\
\hline & SOF/DAC* & $12 \mathrm{~W}$ & SOF/DAC* & $12 W$ \\
\hline \multirow[t]{3}{*}{3} & GLE/PIB* & $8 W$ & GLE/PIB* & $8 W$ \\
\hline & SOF/VEL $L^{*}$ & $12 \mathrm{~W}$ & SOF/VEL* & $12 W$ \\
\hline & SOF/DAC* & $12 \mathrm{~W}$ & SOF/DAC* & $12-24 \mathrm{~W}$ \\
\hline \multirow[t]{4}{*}{$5 / 6$} & GLE/PIB* & $8 W$ & GLE/PIB* & $8 W$ \\
\hline & SOF/VEL & $12 \mathrm{~W}$ & SOF/VEL* & $12 W$ \\
\hline & SOF/DAC* & $12 \mathrm{~W}$ & SOF/DAC* & $12 \mathrm{~W}$ \\
\hline & LDV/SOF & $12 \mathrm{~W}$ & LDV/SOF & $12 \mathrm{~W}$ \\
\hline
\end{tabular}

DAA, direct acting antiviral agent; $H C V$, hepatitis $C$ virus; GLE, glecaprevir; PIB, pibrentasvir; W, weeks; SOF, sofosbuvir; VEL, velpatasvir; DAC, daclatasvir; LDA, ledipasvir; EBR, elbasvir; GZR, grazoprevir.

*Pan-genotypic options. Sofosbuvir/velpatasvir/voxilaprevir is a pangenotypic option for salvage of those who do not achieve sustained virologic responce.

having patients return for clinic visits or obtain monitoring blood draws. Populations that may require more careful monitoring include those with cirrhosis, end-stage renal disease, HCV/HIV or HCV/hepatitis B virus (HBV) coinfection, those with hepatocellular carcinoma, and those who have undergone solid organ transplantation yet many of these populations can be managed by those who directly care for these populations with guidance from hepatitis C specialists.

\section{Care models to eliminate hepatitis C}

Successful treatment and elimination of hepatitis $C$ and meeting 2030 WHO guidelines will only occur by reducing stigma and providing universal access to treatment for those diagnosed with hepatitis C. In addition, efforts to engage high-risk populations who do not routinely seek or have access to health care will be important. Historically, those with untreated opioid use disorder or PWIDs were not treated with interferon-based therapies yet global control of hepatitis C will only occur when models of care for this population are refined to ensure successful diagnosis and treatment in this population. In the DAA era, high sustained response rates were reported in a seminal study for the treatment of hepatitis $\mathrm{C}$ in those receiving opiate agonist therapy with reported sustained response rates above $91.5 \%$. Importantly, relapse rates were relatively low with a 2-year follow-up. ${ }^{27}$ These results have been confirmed in a recent meta-analysis that examined the sustained response rates in 1,702 individuals on opiate substitution therapy (OST) and 538 PWID patients with pooled SVR rate of $90 \%$ for the OST population and pooled SVR rate of $88 \%$ in the PWID population, not different than control populations. Moreover no differences were seen between these populations and controls in adherence and discontinuation rates with reinfection rates ranging from 0 to 12.5 per 100 person years. ${ }^{28}$

Sexual transmission of hepatitis $\mathrm{C}$ in MSM has been responsible for multiple outbreaks of hepatitis $C$. The incidence of hepatitis $C$ in HIV-infected MSM has been estimated to be 6.35/1,000 person-years. ${ }^{29} \mathrm{An}$ increased HCV incidence have been associated with the recent emerging use of chemsex (also known as party and play) which includes crystal methamphetamine, gamma-hydroxybutyrate, or mephedrone, with phosphodiesterase-5 inhibitors before or during sex. ${ }^{30}$ Behavioral factors like serosorting; which is sex between the same HIV status partners and higher rates of anal sex without condoms by HIV-infected men may explain why HIV-infected MSM have higher rates of HCV compared to uninfected MSM. ${ }^{30}$ With the use of pre-exposure prophylaxis (PrEP), HIV-infected men have been reported to become infected with HCV strains known to be found in HIV sexual transmission networks. The use of PrEP also places these individuals at higher risk of HCV by unprotected sexual intercourse with HCV-infected MSM. ${ }^{31}$ Treatment as prevention with universal access to DAA therapy has been shown to reduce the incidence of acute HCV in this population. A recent report from the Netherlands examined the incidence of acute hepatitis $C$ at 17 Dutch HIV centers prior to and after the introduction of universal access to DAA treatment. With rapid uptake in the HIV positive MSM population, a significant reduction in the incidence rate ratio of acute HCV was noted one year after universal access to DAA therapy was made available that differed from the incidence rate ratios of other sexually transmitted diseases such as gonorrhea which rose during the same period. ${ }^{32}$

\section{Emerging models to treat hepatitis C}

Future strategies to treat HCV will involve creating models of care that can engage individuals who have sporadic contact with the healthcare system, with point of care diagnosis and confirmation of active viremia, and subsequent linkage for staging of dis- 
Our approach to hepatitis $C$ must evolve to a decentralized model of care

\begin{tabular}{|c|c|c|c|}
\hline $\begin{array}{l}\text { Improved access to } \\
\text { point of care HCV } \\
\text { testing } \\
\text { Improved education } \\
\text { about testing } \\
\text { Prompts for testing }\end{array}$ & $\begin{array}{l}\text { Access to HCV care } \\
\text { providers } \\
\text { Improved access to care } \\
\text { (universal access) } \\
\text { Co-localized testing and } \\
\text { treatment facilities }\end{array}$ & $\begin{array}{l}\text { Education about } \\
\text { importance of treatment } \\
\text { Psychological and } \\
\text { harm reduction services } \\
\text { for comorbid conditions } \\
\text { Resources for primary care } \\
\text { to treat HCV }\end{array}$ & $\begin{array}{l}\text { Treatment of opioid addiction } \\
\text { Psychological and } \\
\text { harm reduction services } \\
\text { for comorbid conditions } \\
\text { Directly observed therapy }\end{array}$ \\
\hline
\end{tabular}

Figure 2. Our approach to hepatitis C should evolve to a decentralized model of care. $\mathrm{HCV}$, hepatitis C virus.

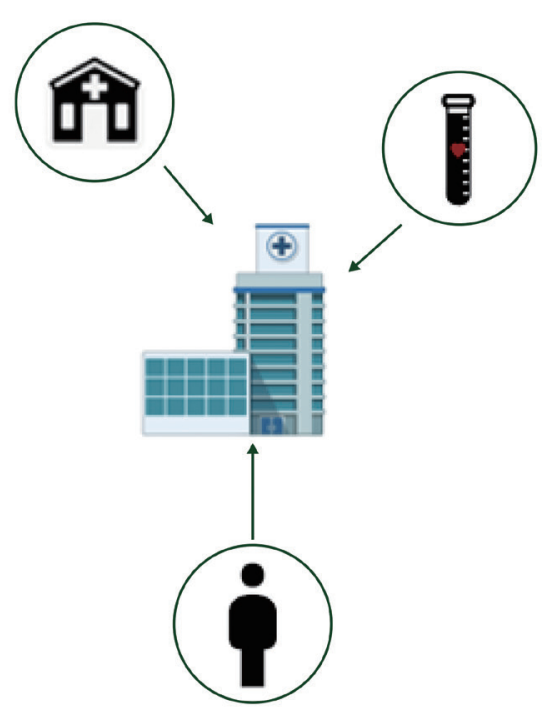

Centralized testing for diagnosis of hepatitis $C$ with linkage to care

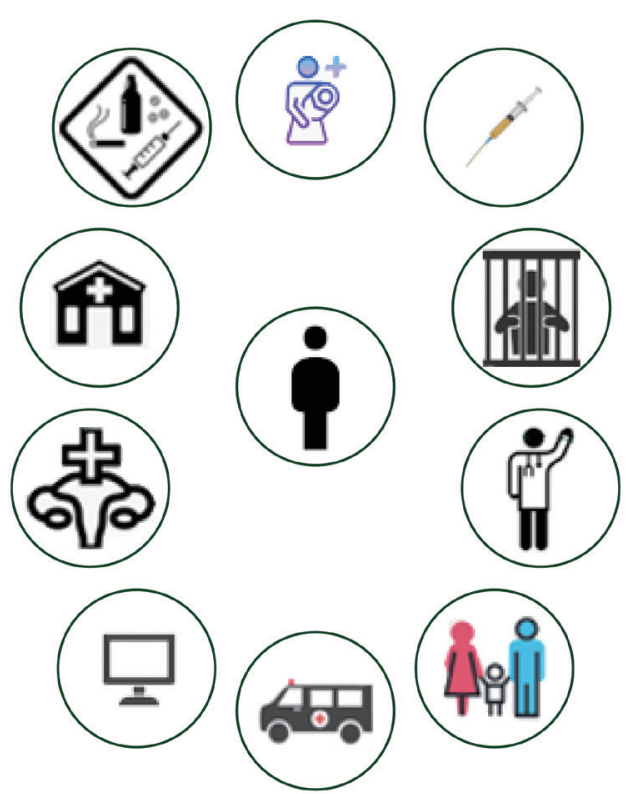

Decentralized testing for diagnosis of hepatitis $C$ with linkage to care

Figure 3. To eliminate hepatitis $C$, we will need to transition to decentralized diagnosis and treatment of hepatitis $C$ by bringing hepatitis $C$ care to primary care clinics, rehabilitation centers, needle syringe programs, prisons, community health centers, sexual health centers, emergency/urgent care centers in addition to expanded use of telehealth and mobile health care units.

ease, and appropriate treatment (Fig. 2). These evolving strategies have led to the transition to a decentralized approach, where individuals present for medical care at primary care and other clinics, receive diagnostic testing, evaluation, and treatment for hepatitis C with DAA therapy (Fig. 3) rather than be referred to a center specializing in the treatment of HCV. These clinics may collaborate with specialists who can ensure that individuals with HCV are properly evaluated and that clinic staff are properly trained to provide both diagnostic testing and treatment for hepatitis $\mathrm{C}$.

Incorporation of $\mathrm{HCV}$ treatment into clinics that care for high risk patients including those with opioid use disorder will be an important strategy and emerging data has given us insights into strategies that may be effective. One recently reported trial at a harm reduction center reported results in 100 patients with hepatitis $C$ and opioid use disorder who received a 12 week course of DAA therapy and were offered buprenorphine therapy at initiation of HCV treatment. ${ }^{33}$ The highest sustained response rates were seen in individuals who initiated buprenorphine and were retained in treatment with an overall SVR rate of $92 \%$, which was superior to the SVR rate seen in those whose buprenorphine was discontinued or who never received buprenorphine, emphasizing the need for harm reduction measures being combined with treatment of HCV. Receipt of buprenorphine at the time of treatment was also associated with lower HIV risk-taking behavior scores 
and lower rates of opioid overdose.

Treatment as prevention is evolving as a major strategy for the treatment of HCV. Iceland initiated the Treatment As Prevention For Hepatitis C Program in 2016 with the aim of treating every patient with HCV free of charge. Within 3 years of initiation, 98\% of those confirmed with HCV RNA positive testing initiated DAA therapy with an overall SVR rate of $89 \% .{ }^{34}$ While there are unique aspects regarding Iceland including its small population with just one hospital providing care to these individuals, their findings demonstrate that a strong commitment and scale up to testing with linkage to treatment can lead to high rates of diagnosis and treatment.

The use of telehealth to train healthcare providers to care for and treat those with HCV will play a major role in hepatitis $C$ elimination. In the USA, the creation of Extension for Community Healthcare Outcomes (ECHO) model of care for hepatitis $\mathrm{C}$ in has allowed access to treatment for hepatitis $\mathrm{C}$ and other diseases in areas with a shortage of specialists. The ECHO program uses a multidisciplinary hub- team to train providers to care for and treat those with hepatitis $C$ while achieving sustained response rates that are equivalent to specialist based care. A recent report noted there are over 25 HCV ECHO hubs in the USA and at least 16 international HCV ECHO programs worldwide. ${ }^{35}$ Similar successful telehealth programs have been implemented in countries across the world and telehealth will likely play a greater role in delivering health care across all disease states.

\section{Recently reported initiatives}

Multiple countries and regions of the world have committed to eliminate hepatitis $C$ with development of action plans to educate the public about $\mathrm{HCV}$, increase diagnosis, and improve linkage to care. Two recent reports highlight this strategy can be successfully deployed on a large scale, to allow those without routine access to specialist care to receive treatment for HCV. India created a national action plan to eliminate viral hepatitis and the initial report from this effort has been reported which consisted of training primary care providers from university and district hospitals through e-learning and e-courses to provide algorithm based hepatitis $C$ treatment using generic DAA regimens. Primary care providers were supervised via telehealth with assessments being done locally, and noninvasive markers were used to assess fibrosis. ${ }^{36}$ This largely rural population (80.5\%) included those with cirrhosis (14.8\% as assessed by APRI, FIB-4 or fibroscan) with the majority infected with genotype $3 \mathrm{HCV}$.
An interim report notes that of 48,088 individuals initially enrolled, $36,250(75.4 \%)$ completed treatment thus far with a per protocol sustained response rate of $91.6 \%$, and sustained response rate of $84.4 \%$ in individuals who had treatment interruptions.

The prevalence of HCV in Egypt has been amongst the highest of all countries due to prior treatment of schistosomiasis with unsafe injections. In 2018, Egypt initiated a nationwide program to screen for and treat hepatitis $C^{37}$ In this ambitious program, nearly 50 million individuals were screened using a WHO approved rapid diagnostic test. The effort was comprehensive with screening occurring in all primary and rural health units, all Egyptian health insurance managed clinics, hospital and police hospitals, and all youth centers. Mobile screening teams were also deployed to crowded areas on special occasions including churches, mosques, soccer stadiums and picnic areas. Nearly 50 million individuals participated in the screening program, which found an overall seropositivity rate of $4.6 \%$ with higher rates in the Nile Delta. Three fourths $(76.5 \%)$ of the individuals with positive HCV rapid diagnostic test had detectable viremia of which $91.8 \%$ of these individuals initiated treatment with generic DAA therapy. An interim per protocol SVR rate was recently reported at $98.8 \%$ $(381,491 / 386,103)$. This large-scale project highlights several important components of future strategies for treatment of hepatitis C. The government created a national education plan with advertising on television and radio, combined with newspaper advertisements, billboard campaigns, and text messaging. There was large deployment of screening teams that comprised of a physician, nurse and data entry person with widespread availability for patients during the screening phase ( 12 hours per day 7 days per week). Pricing for diagnostic testing including antibody and confirmatory PCR testing was negotiated to make the screening program affordable with a negotiated cost of 0.58 USD for the rapid diagnostic antibody test and 4.80 USD per PCR test including use of PCR machines with linkage to a central database. This successful model of care used a decentralized approach, with universal screening in all healthcare clinics and universal access to DAA therapy and Egypt, a low to middle income country which had the highest prevalence of hepatitis $C$, may be the first country that achieves elimination of hepatitis $\mathrm{C}$.

Though the prevalence of HCV is less than HBV in Asia, the infrastructure that has emerged to care for those with hepatitis $B$ can be used as a foundation throughout Asia to achieve hepatitis C elimination by 2030. In Asia, Taiwan has reported that they are on track to eliminate hepatitis C. ${ }^{38}$ The Taiwan Hepatitis C Policy 
Guideline 2018-2025 policy implemented in 2019 will provide 1.7 billion USD over 8 years to lower barriers of access to care and improve screening strategies and care models for high-risk populations. Similar to other successful programs, there is a government effort to educate the public about hepatitis $C$, improving health literacy to prevent new infection and reinfection. Based on modeling data, it is now projected that Taiwan will meet WHO guidance by $2025 .{ }^{39}$ In Korea the prevalence of hepatitis $C$ has been estimated to be $0.44 \%$ and hepatitis $\mathrm{C}$ has been designated as a group 3 communicable disease with mandatory sentinel surveillance of new cases. ${ }^{40}$ Currently there is no universal screening program to identify undiagnosed hepatitis $\mathrm{C}$ cases in Korea and it has been estimated that up to three quarters of individuals may be unaware of their infection. Those who are diagnosed with hepatitis C present to specialists to receive DAA therapy. A recent paper examined multiple strategies to meet WHO 2030 elimination targets, with strategies to meet elimination targets by 2025 or 2030 leading to significant cost savings though initial capital investments would be required. However, to reach elimination targets by 2025, existing diagnostic and treatment practices must be increased nearly 4.5-fold and 3-fold, respectively. Finally, numerous successful reports of elimination projects have been reported in Rwanda, Mongolia, and the Republic of Georgia amongst others. ${ }^{41-43}$

With the high prevalence of HCV in correction facilities, this population will require new models of care to diagnose, link to and provide appropriate DAA therapy. This will require universal screening programs with all confirmed HCV-positive prisoners then being offered treatment during custody or after release from prisons. A recent two-phase case study of HCV care pathway was implemented in the London prison system by offering dried blood spot testing to new arrivals at their secondary health check and highlights the challenges with treating this population. ${ }^{44}$ Prisoners with active HCV infection finished disease stratification tests and were reviewed weekly at a hospital-based team meeting to decide management. In phase-2, the pathway was redesigned to of- fer opt-out blood borne virus testing with treatment referral of $\mathrm{HCV}$-positive prisoners. As a result, linkage to HCV care improved though was still not ideal with $38.9 \%$ of incarcerated individuals being treated or referred during the second phase. Elimination of $\mathrm{HCV}$ in prisons will require local service configuration to ensure high uptake of testing, with all HCV-positive cases then being offered treatment during custody or referral to treatment after release.

\section{FUTURE TREATMENTS FOR HEPATITIS C}

With our current DAA therapies, sustained response or cure is achieved in over $90 \%$ of individuals and thus the need for novel therapies for HCV is not urgent. However, with the goal of elimination of hepatitis $C$ now being pursued, it is important to note that the majority of infectious diseases such as smallpox or polio that have been successfully eliminated or eradicated have included the development of an effective vaccine. A significant challenge for development of an HCV vaccine is the genetic diversity of the virus. Different genotypes of HCV strains differ in approximately $30 \%$ of their amino acids, and different HCV subtypes in each genotype differ in approximately $15 \%$ of their amino acids. ${ }^{45}$ The HCV can generate a diverse quasispecies of related but genetically different viral variants within each infected individual by immune selection as well as the error-prone viral polymerase. Strategies to develop an HCV vaccine have included vaccines to elicit $T$ cell responses, and vaccines to generate sufficient neutralizing antibody titers including the development of broadly neutralizing antibodies. ${ }^{46}$ However, to date, no strategy has been proven to be effective in preventing hepatitis $\mathrm{C}$ infection. One recent clinical trial evaluating the experimental hepatitis $\mathrm{C}$ prime boost vaccine AdCh3NSmut1/MVA-NSmut was not found to be effective in preventing chronic HCV in a randomized trial of high risk individuals (https://www.niaid.nih.gov/news-events/trial-evaluating-experimental-hepatitis-c-vaccine-concludes). Nonetheless, develop-

Universal care pathway

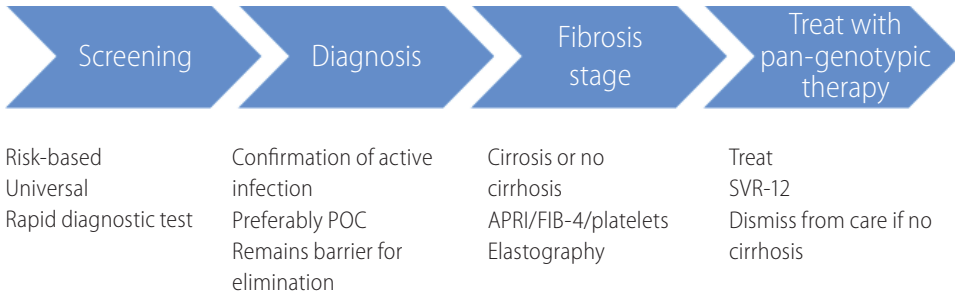

http://www.e-cmh.org
Figure 4. A universal care pathway of hepatitis $C$ can be constructed to reach elimination goals. POC, point of care; APRI, aspartate aminotransferase-platelets ratio index; FIB-4, fibrosis-4; SVR, sustained virologic response. 
ment of a successful hepatitis $C$ vaccine would be an important advance in efforts toward global control of HCV.

Hepatitis C infection is straightforward to treat and difficult to eliminate. We already have the tools to successfully treat the vast majority of individuals who are diagnosed with HCV. However, elimination of HCV will require a strong commitment from governments and payors to provide funding for education, diagnosis, and treatment. The most important gap in the cascade of care that must be addressed is to improve the screening and diagnosis rate for those infected with HCV. Risk-based screening of targeted populations, risk behavior screening, and universal screening will all have a role in finding the large pool of individuals who have yet to be diagnosed with hepatitis $C$. Whereas diagnostic testing for exposure to HCV is inexpensive and can be performed as a rapid diagnostic test, confirmation of active viremia remains a challenge in many parts of the world and the point-of-care test to confirm active infection remains an important challenge. Colocalization of hepatitis $C$ diagnosis and treatment in all types of healthcare clinics that care for patients who are at risk for $\mathrm{HCV}$ is an evolving and essential strategy that will allow the large pool of individuals yet to be diagnosed the opportunity to link to care for their hepatitis C. Simplified treatment algorithms will improve access to treatment. These decentralized approaches have been implemented successfully world-wide as part of elimination strategies allowing those infected with HCV local access to treatment (Fig. 4). While a vaccine to prevent HCV would be an ideal addition to our therapeutic armamentarium the results to date have not yet proven successful. There are success stories of disease eradication without vaccination such as the treatment of river blindness and we should press forward across the world with our efforts to successfully diagnose, treat and eliminate hepatitis $C$ by 2030.

\section{Authors' contribution}

Omar Alshuwaykh: Literature search, drafting of manuscript and critical revisions; Paul Kwo: Literature search, drafting of manuscript and critical revisions

\section{Conflicts of Interest}

Potential competing interests Paul Y. Kwo: Abbvie, Gilead (Advisory Board); BMS, Gilead, Target Registries (Grant Support); Syneos (DSMB)

No potential competing interests for Omar Alshuwaykh

\section{REFERENCES}

1. Kuo G, Choo QL, Alter HJ, Gitnick GL, Redeker AG, Purcell RH, et al. An assay for circulating antibodies to a major etiologic virus of human non-A, non-B hepatitis. Science 1989;244:362-364.

2. Choo QL, Kuo G, Weiner AJ, Overby LR, Bradley DW, Houghton M. Isolation of a cDNA clone derived from a blood-borne non-A, non-B viral hepatitis genome. Science 1989;244:359-362.

3. Appelbaum E, Kalkstein M. Artificial transmission of viral hepatitis among intravenous diacetylmorphine addicts. J Am Med Assoc 1951;147:222-224.

4. Davis GL, Balart LA, Schiff ER, Lindsay K, Bodenheimer HC Jr, Perrillo RP, et al. Treatment of chronic hepatitis $C$ with recombinant interferon alfa. A multicenter randomized, controlled trial. N Engl J Med 1989;321:1501-1506.

5. Hoofnagle JH, Seeff LB. Peginterferon and ribavirin for chronic hepatitis C. N Engl J Med 2006;355:2444-2451.

6. Falade-Nwulia O, Suarez-Cuervo C, Nelson DR, Fried MW, Segal JB, Sulkowski MS. Oral direct-acting agent therapy for hepatitis $C$ virus infection: a systematic review. Ann Intern Med 2017;166:637-648.

7. Polaris Observatory HCV Collaborators. Global prevalence and genotype distribution of hepatitis C virus infection in 2015: a modelling study. Lancet Gastroenterol Hepatol 2017;2:161-176.

8. World Health Organization (WHO). Global health sector strategy on viral hepatitis 2016-2021. Towards ending viral hepatitis. Geneva: WHO, 2016.

9. Lazarus JV, Roel E, Elsharkawy AM. Hepatitis C virus epidemiology and the impact of interferon-free hepatitis C virus therapy. Cold Spring Harb Perspect Med 2020;10:a036913.

10. Dillon JF, Lazarus JV, Razavi HA. Urgent action to fight hepatitis $C$ in people who inject drugs in Europe. Hepatol Med Policy 2016;1:2.

11. Zhang AY, Shrum S, Williams S, Petnic S, Nadle J, Johnston $H$, et al. The changing epidemiology of candidemia in the United States: injection drug use as an increasingly common risk factor-active surveillance in selected sites, United States, 2014-2017. Clin Infect Dis 2020;71:1732-1737.

12. Grebely J, Dore GJ. Can hepatitis C virus infection be eradicated in people who inject drugs? Antiviral Res 2014;104:62-72.

13. Ryerson $A B$, Schillie $S$, Barker $L K$, Kupronis BA, Wester C. Vital signs: newly reported acute and chronic hepatitis C cases - United States, 2009-2018. MMWR Morb Mortal Wkly Rep 2020;69:399-404.

14. Dolan K, Wirtz AL, Moazen B, Ndeffo-Mbah M, Galvani A, Kinner $S A$, et al. Global burden of HIV, viral hepatitis, and tuberculosis in prisoners and detainees. Lancet 2016;388:1089-1102.

15. Kinner SA, Snow K, Wirtz AL, Altice FL, Beyrer C, Dolan K. Agespecific global prevalence of hepatitis $B$, hepatitis $C$, HIV, and tuberculosis among incarcerated people: a systematic review. J Adolesc Health 2018;62:S18-S26. 
Omar Alshuwaykh, et al. Current and future strategies for $\mathrm{HCV}$

16. Flemming JA, Kim WR, Brosgart CL, Terrault NA. Reduction in liver transplant wait-listing in the era of direct-acting antiviral therapy. Hepatology 2017;65:804-812.

17. Dore GJ, Matthews GV. Universal screening for hepatitis C virus infection should be linked to universal treatment access. Nat Rev Gastroenterol Hepatol 2020;17:321-322.

18. Graham CS, Trooskin S. Universal screening for hepatitis C virus infection: a step toward elimination. JAMA 2020;323:936-937.

19. US Preventive Services Task Force; Owens DK, Davidson KW, Krist AH, Barry MJ, Cabana $M$, et al. Screening for hepatitis C virus infection in adolescents and adults: US preventive services task force recommendation statement. JAMA 2020;323:970-975.

20. European Association for the Study of the Liver. EASL recommendations on treatment of hepatitis C 2018. J Hepatol 2018;69:461-511.

21. Omata M, Kanda T, Wei L, Yu ML, Chuang WL, Ibrahim A, et al. APASL consensus statements and recommendations for hepatitis C prevention, epidemiology, and laboratory testing. Hepatol Int 2016;10:681-701.

22. World Health Organization (WHO). WHO guidelines on hepatitis B and C testing. Geneva: WHO, 2017.

23. Mathur $P$, Kottilil $S$. Hepatitis $C$ core antigen testing: still an effective diagnostic method for global elimination of hepatitis C. Clin Infect Dis 2020;70:674-675.

24. Bethea ED, Chen Q, Hur C, Chung RT, Chhatwal J. Should we treat acute hepatitis C? A decision and cost-effectiveness analysis. Hepatology 2018;67:837-846.

25. Ghany MG, Morgan TR; AASLD-IDSA Hepatitis C Guidance Panel. Hepatitis C guidance 2019 update: American Association for the Study of Liver Diseases-infectious diseases society of America recommendations for testing, managing, and treating hepatitis $C$ virus infection. Hepatology 2020;71:686-721.

26. Dieterich DT. A simplified algorithm for the management of hepatitis C infection. Gastroenterol Hepatol (N Y) 2019;15(5 Suppl 3):1-12.

27. Dore GJ, Altice F, Litwin AH, Dalgard O, Gane EJ, Shibolet O, et al. Elbasvir-Grazoprevir to treat hepatitis $\mathrm{C}$ virus infection in persons receiving opioid agonist therapy: a randomized trial. Ann Intern Med 2016;165:625-634

28. Graf C, Mücke MM, Dultz G, Peiffer KH, Kubesch A, Ingiliz P, et al. Efficacy of direct-acting antivirals for chronic hepatitis $C$ virus infection in people who inject drugs or receive opioid substitution therapy: a systematic review and meta-analysis. Clin Infect Dis 2020;70:2355-2365.

29. Jin $F$, Matthews GV, Grulich AE. Sexual transmission of hepatitis $C$ virus among gay and bisexual men: a systematic review. Sex Health 2017;14:28-41.

30. Pufall EL, Kall M, Shahmanesh M, Nardone A, Gilson R, Delpech $V$, et al. Sexualized drug use ('chemsex') and high-risk sexual behaviours in HIV-positive men who have sex with men. HIV Med
2018;19:261-270.

31. Hoornenborg E, Achterbergh RCA, Schim van der Loeff MF, Davidovich $U$, Hogewoning A, de Vries HJC, et al. MSM starting preexposure prophylaxis are at risk of hepatitis $C$ virus infection. AIDS 2017;31:1603-1610.

32. Boerekamps A, van den Berk GE, Lauw FN, Leyten EM, van Kasteren $M E$, van Eeden $A$, et al. Declining hepatitis $C$ virus (HCV) incidence in Dutch human immunodeficiency virus-positive men who have sex with men after unrestricted access to HCV therapy. Clin Infect Dis 2018;66:1360-1365.

33. Rosenthal ES, Silk R, Mathur P, Gross C, Eyasu R, Nussdorf $L$, et al. Concurrent initiation of hepatitis $C$ and opioid use disorder treatment in people who inject drugs. Clin Infect Dis 2020;71:1715-1722.

34. Scott N, Ólafsson S, Gottfreðsson M, Tyrfingsson T, Rúnarsdóttir V, Hansdottir I, et al. Modelling the elimination of hepatitis $C$ as a public health threat in Iceland: a goal attainable by 2020. J Hepatol 2018;68:932-939.

35. Arora S. Project ECHO: democratising knowledge for the elimination of viral hepatitis. Lancet Gastroenterol Hepatol 2019;4:91-93.

36. Dhiman RK, Grover GS, Premkumar M, Taneja S, Duseja A, Arora S, et al. Decentralized care with generic direct-acting antivirals in the management of chronic hepatitis $C$ in a public health care setting. J Hepatol 2019;71:1076-1085.

37. Waked I, Esmat G, Elsharkawy A, El-Serafy M, Abdel-Razek W, Ghalab $R$, et al. Screening and treatment program to eliminate hepatitis C in Egypt. N Engl J Med 2020;382:1166-1174.

38. Chen DS. Taiwan commits to eliminating hepatitis $C$ in 2025. Lancet Infect Dis 2019;19:466-467.

39. Wu GH, Pwu RF, Chen SC, Chen DS. Taiwan is on track of accelerating hepatitis C elimination by 2025. Liver Int 2020;40:1506-1507.

40. Sinn DH, Cho EJ, Kim JH, Kim DY, Kim YJ, Choi MS. Current status and strategies for viral hepatitis control in Korea. Clin Mol Hepatol 2017;23:189-195.

41. Hagan LM, Kasradze A, Salyer SJ, Gamkrelidze A, Alkhazashvili M, Chanturia $G$, et al. Hepatitis $C$ prevalence and risk factors in Georgia, 2015: setting a baseline for elimination. BMC Public Health 2019;19(Suppl 3):480.

42. Tsertsvadze T, Gamkrelidze A, Chkhartishvili N, Abutidze A, Sharvadze $L$, Kerashvili $V$, et al. Three years of progress toward achieving hepatitis C elimination in the country of Georgia, April 2015-March 2018. Clin Infect Dis 2020;71:1263-1268.

43. Umutesi G, Shumbusho F, Kateera F, Serumondo J, Kabahizi J, Musabeyezu $E$, et al. Rwanda launches a 5-year national hepatitis C elimination plan: a landmark in sub-Saharan Africa. J Hepatol 2019;70:1043-1045.

44. Connoley D, Francis-Graham S, Storer M, Ekeke N, Smith C, Macdonald $\mathrm{D}$, et al. Detection, stratification and treatment of hepatitis C-positive prisoners in the United Kingdom prison estate: develop- 


\section{CLINCAL and MOLECULAR
HEPATOLOGY}

ment of a pathway of care to facilitate the elimination of hepatitis $C$ in a London prison. J Viral Hepat 2020;27:987-995.

45. Smith DB, Bukh J, Kuiken C, Muerhoff AS, Rice CM, Stapleton JT, et al. Expanded classification of hepatitis $C$ virus into 7 genotypes and 67 subtypes: updated criteria and genotype assignment web resource. Hepatology 2014;59:318-327.

46. Bailey JR, Barnes E, Cox AL. Approaches, progress, and challenges to hepatitis $C$ vaccine development. Gastroenterology 2019;156:418-430.
47. World Health Organization (WHO). Progress report on access to hepatitis c treatment: focus on overcoming barriers in low- and middle-income countries. March 2018. WHO web site, <https:// apps.who.int/iris/bitstream/handle/10665/260445/WHO-CDS-HIV18.4-eng.pdf? sequence=1>. Accessed 1 Aug 2020.

48. World Health Organization (WHO). In vitro diagnostics and laboratory technology. WHO web site, <https://www.who.int/diagnostics_ laboratory/evaluations/pq-list/hcv/public_report/en/>. Accessed 1 Aug 2020. 